\title{
Clinical effect of a dentifrice containing three kinds of bactericidal ingredients on periodontal disease: a pilot study in patients undergoing supportive periodontal therapy
}

\author{
Daichi Kita ${ }^{1 \dagger}$, Takashi Kinumatsu ${ }^{1,2+}$, Atsushi Yokomizo ${ }^{3}$, Miki Tanaka $^{3}$, Masahiro Egawa ${ }^{1}$, Asako Makino-Oi ${ }^{1}$,
} Sachiyo Tomita ${ }^{1}$ and Atsushi Saito ${ }^{1,4^{*}}$ (1)

\begin{abstract}
Objective: This study aimed to evaluate clinically the effect of a novel dentifrice containing three kinds of bactericidal ingredients on periodontal disease.

Results: This was a single-arm, prospective clinical study that enrolled patients with periodontitis undergoing supportive periodontal therapy. Periodontal examination, microbiological testing of saliva samples, and evaluation of inflammatory markers (IL-1 $\beta$, IL-6, IL-8, TNF- $\alpha$ ) in gingival crevicular fluid were performed. After 4 weeks of the use of test dentifrice, these parameters were re-evaluated. The use of dentifrice was also subjectively evaluated by clinicians and participants. Among 30 participants, there were significant improvements in the periodontal and microbiological parameters, and the level of interleukin-1 $\beta$ in the gingival crevicular fluid, following the use of the test dentifrice. In clinicians' subjective evaluation of the overall usefulness of the dentifrice, 'mild' and 'moderate' improvement accounted for $83 \%$ of the total responses. In the participants' subjective evaluation, the majority indicated their experience of the use as favorable. Within the limitations of this study, it is suggested that the progression of periodontal disease during the supportive periodontal therapy can be prevented by the use of the test dentifrice.

Trial registration UMIN Clinical Trials Registry (UMIN-CTR) 000023175. Date of formal registration: July 14, 2016 (https:// upload.umin.ac.jp/cgi-open-bin/ctr/ctr_view.cgi?recptno=R000026716)
\end{abstract}

Keywords: Periodontal disease, Supportive periodontal therapy, Dentifrice, Bactericidal ingredients

\section{Introduction}

Periodontal diseases are the major cause of loss of teeth $[1,2]$. The destruction of periodontal tissue due to the diseases is thought to be caused mainly by inflammation induced by dental plaque consisting of periodontopathogenic bacteria [2]. To prevent periodontal diseases, mechanical plaque control by toothbrush is the most fundamental and effective. However, the mechanical means alone have limitation for the thorough plaque control [3].

\footnotetext{
*Correspondence: atsaito@tdc.ac.jp

${ }^{\dagger}$ Daichi Kita and Takashi Kinumatsu contributed equally to this work ${ }^{1}$ Department of Periodontology, Tokyo Dental College, Tokyo, Japan Full list of author information is available at the end of the article
}

In everyday self-care, dentifrices are used as a part of plaque control. It has been reported that the use of dentifrices containing antimicrobial ingredients can enhance the preventive effect on periodontal disease $[4,5]$. Medical ingredients such as sodium lauroylsarcosine, isopropyl methylphenol and cetylpyridinium chloride have been shown to be effective in preventing periodontal diseases $[4,5]$. However, the effects of combining those ingredients on the prevention of periodontal disease progression have not been clarified.

This study aimed to evaluate the effect of a dentifrice containing the three kinds of bactericidal ingredients on periodontal disease in patients undergoing supportive periodontal therapy (SPT). 


\section{Main text \\ Methods \\ Study design}

This was a prospective, single-arm clinical study. Participants were recruited from patients with slight or moderate chronic periodontitis [6] who have been receiving SPT at Tokyo Dental College Suidobashi Hospital (Tokyo, Japan) or Tokyo Dental College Chiba Hospital (Chiba, Japan). Sample size was set as 29 patients based on the difference in gingival index (GI) in the previous study using dentifrice [7]; effect quantity: 0.24, standard deviation: 0.5 , $\alpha$-value: 0.05 , detection power: 0.80 .

\section{Inclusion and exclusion criteria}

Written informed consent was obtained from all participants. To qualify, patients were required to have a minimum of 20 natural teeth, having at least one site with probing depth (PD) $\geq 4 \mathrm{~mm}$ and GI score of 1 or 2. Exclusion criteria were the presence of uncontrolled systemic diseases, smokers, allergy to the dentifrice ingredients, and current pregnancy or lactation. Those with history of active periodontal treatment including scaling and root planning, antimicrobial therapy within 3 months, and contraindications for general dental interventions were excluded. Those who had been already using a previous version of the test dentifrice (Clean Dental ${ }^{\circledR}$, Daiichi Sankyo Healthcare, Tokyo, Japan) are also excluded. The participants received no medication that could affect their periodontal conditions, such as antimicrobial agents or anti-inflammatory drugs, for at least 3 months prior to the study.

\section{Periodontal examination}

At baseline, trained, calibrated examiners recorded the following: number of sites with gingival swelling, redness, plaque index (PII), GI, gingival recession, PD and clinical attachment level (CAL) essentially as described previously [8]. These parameters were also recorded at 2 and 4 weeks.

\section{Microbiological testing}

Saliva samples were collected according to the manufacturer's instructions and immediately transferred into sampling tubes supplied in a commercial kit (GC, Tokyo, Japan) and sent to a commercial testing laboratory (GC Oral Check Center, Tokyo, Japan: http://www.gcoc.jp/). The numbers (copies) of periodontal pathogens (Porphyromonas gingivalis, Aggregatibacter actinomycetemcomitans, Treponema denticola, Tannerella forsythia, Prevotella intermedia) and the total bacteria in saliva were measured by using real-time polymerase chain reaction method.

\section{Assessment of inflammatory markers}

Sampling of gingival crevicular fluid (GCF) was performed by inserting a paper strip into the gingival sulcus of one tooth with a deep pocket. Samples were sent to a commercial testing laboratory (GeneticLab, Sapporo, Japan). Measurement of inflammatory markers (IL-1 $\beta$, IL-6, IL-8, TNF- $\alpha$ ) in GCF was performed by the laboratory. Briefly, the protein was extracted from the paper strip and the total protein content $(\mathrm{mg} / \mathrm{mL})$ in the extraction solution was measured by a colorimetric method. After labeling of each inflammatory marker, the amount in the extraction solution $(\mathrm{pg} / \mathrm{mL})$ was calculated by measuring the fluorescence intensity. Finally, the amount of each inflammatory marker $(\mathrm{pg} / \mathrm{mg})$ to the total protein mass of $1 \mathrm{mg}$ was calculated by dividing the amount of the inflammatory marker in GCF $(\mathrm{pg} / \mathrm{mL})$ by the total protein content $(\mathrm{mg} / \mathrm{mL})$.

\section{Use of test dentifrice}

A novel dentifrice (OC 1510, provisional name: Clean Dental $^{\circledR}$ product under development, Daiichi Sankyo Healthcare) containing 10 medicinal ingredients including 3 bactericidal agents (sodium lauroylsarcosine, isopropyl methylphenol and cetylpyridinium chloride) and 2 anti-inflammatory agents ( $\beta$-glycyrrhetinic acid and $\varepsilon$-aminocaproic acid) was used for the study. Participants were asked to use $1 \mathrm{~g}$ of the test dentifrice and brush with the scrubbing method 3 times/day after meals for 4 weeks. A standard toothbrush (Dent EX Systema 44M, Lion, Japan) was supplied to each participant. A leaflet describing the toothbrushing method using the test dentifrice was distributed to each participant. In order to monitor compliance, they were asked to record the use of dentifrice.

\section{Subjective evaluation of overall usefulness by clinicians}

At the end of the study, supervising clinicians evaluated the participants' overall usefulness of the test dentifrice relative to baseline conditions, using a Likert scale: 5; marked improvement, 4; moderate improvement, 3 ; mild improvement, 2; no difference, 1: worse.

\section{Subjective evaluation by participants}

A questionnaire survey on the use of test dentifrice was carried out. The questionnaire items are shown in Additional file 1: Table S1.

\section{Statistical analysis}

For statistical analysis, GraphPad InStat version 3.10 (GraphPad Software, Inc. La Jolla, CA) was used. 
Friedman test with Dunn post-hoc test was used, and p-values less than 0.05 were considered statistically significant.

\section{Results}

\section{Demographic information on participants}

In this study, a total of 30 chronic periodontitis patients ( 9 males and 21 females, mean 58.6 years old, range: $32-70$ years old) were enrolled and completed the study.

\section{Periodontal examination}

The results of periodontal examination are shown in Table 1. Significant improvements were observed in gingival redness, PII and GI at 2 weeks and 4 weeks compared to baseline $(\mathrm{p}<0.05)$. At 2 weeks, no significant improvement from baseline was observed in gingival swelling, PD, CAL, and GI $\geq 2$ sites. However, significant improvement was observed at 4 weeks $(\mathrm{p}<0.01)$. In the amount of gingival recession, significant difference from baseline and 2 weeks was noted at 4 weeks $(\mathrm{p}<0.001)$.

Throughout the study, no serious adverse events were reported.

\section{Microbiological findings}

The results of the microbiological testing are shown in Table 2. The copy numbers of T. denticola and T. forsythia

Table 1 Changes in periodontal parameters

\begin{tabular}{lcll}
\hline & Baseline & 2 weeks & 4 weeks \\
\hline Gingival swelling & $0.22 \pm 0.35$ & $0.11 \pm 0.22$ & $0.06 \pm 0.12^{* *}$ \\
Gingival redness & $0.32 \pm 0.41$ & $0.19 \pm 0.33^{*}$ & $0.16 \pm 0.24^{* * *}$ \\
PII & $0.31 \pm 0.32$ & $0.18 \pm 0.18^{* *}$ & $0.17 \pm 0.18^{* *}$ \\
Gl & $0.31 \pm 0.27$ & $0.22 \pm 0.25^{*}$ & $0.17 \pm 0.17^{* * *}$ \\
Gingival recession (mm) & $0.56 \pm 0.50$ & $0.56 \pm 0.52$ & $0.61 \pm 0.54^{* * *},++\dagger$ \\
PD (mm) & $2.24 \pm 0.27$ & $2.17 \pm 0.26$ & $2.10 \pm 0.29^{* * *}$ \\
CAL (mm) & $2.80 \pm 0.51$ & $2.73 \pm 0.58$ & $2.71 \pm 0.54^{* *}$ \\
Number of sites with & $16.33 \pm 15.86$ & $11.70 \pm 12.65$ & $9.10 \pm 10.27^{* * *}$ \\
Gl $\geq 2$ & & & \\
\hline
\end{tabular}

Data shown as mean \pm standard deviation. ${ }^{*} p<0.05$, ${ }^{* *} p<0.01,{ }^{* * *} p<0.001$ vs. baseline, ${ }^{t+t} p<0.001$ vs. 2 weeks were significantly decreased at 4 weeks compared to baseline $(\mathrm{p}<0.05)$. No significant change was observed in other bacteria for 4 weeks. Total copy numbers of bacteria were significantly decreased at 2 weeks compared to baseline $(\mathrm{p}<0.01)$, but no significant difference was observed at 4 weeks.

\section{Inflammatory markers in GCF}

The measurement results of inflammatory markers in GCF are shown in Additional file 2: Table S2. The sample size of IL- 6 was smaller than other cytokines, because the median values of the obtained fluorescence intensity were too low for calculating the concentration in some samples.

Among the four cytokines, a significant reduction from baseline was observed for IL-1 $\beta$ at 2 weeks $(p<0.05)$. No significant difference was observed between 2 weeks and 4 weeks. In other cytokines, no significant change was observed during the test period.

\section{Overall usefulness}

"Mild improvement" was reported by 60\% (18/30) of clinicians, followed by "moderate improvement" $23.3 \%$ (7/30), and "no difference" $16.7 \%$ (5/30).

\section{Subjective evaluation by the participants}

The questionnaire results for the use of test dentifrice are shown in Fig. 1. In response to "feeling after use", 24 (80\%) participants scored 4 (good) or 5 (very good). In response to "perceived effect", 29 (96.7\%) participants scored 4 or 5 . As for "improvement of symptoms", 24 (80\%) participants scored 4 (slight improvement) or 5 (much improvement). In response to "general preference of test dentifrice", 19 (63.3\%) participants scored 4 (somewhat preferred) or 5 (much preferred), while two (6.7\%) scored 2 (somewhat dispreferred). As for "comparison to ordinary use toothpastes", 24 (80\%) of participants scored 5 (much preferred), but 6 (20\%) participants scored 1 (dispreferred). In response to "likelihood of continued use of test dentifrice hereafter", 25 (83.3\%) participants scored 4 (probably) or 5 (definitely), but one (3.3\%)

Table 2 Number of bacteria; log copies (ratio of each bacteria to the total number; $\times 10^{-3} \%$ )

\begin{tabular}{llll}
\hline & Baseline & 2 weeks & 4 weeks \\
\hline Total bacteria & $9.24 \pm 9.08$ & $9.01 \pm 8.92^{* *}$ & $9.04 \pm 8.87$ \\
Porphyromonas gingivalis & $5.89 \pm 6.35(37.48 \pm 95.97)$ & $5.26 \pm 5.55(17.21 \pm 30.54)$ & $5.46 \pm 5.93(20.09 \pm 39.56)$ \\
$\begin{array}{l}\text { Aggregatibacter actinomycetemcomi- } \\
\quad \text { tans }\end{array}$ & $3.06 \pm 3.63(0.12 \pm 0.48)$ & $2.87 \pm 3.37(0.14 \pm 0.61)$ & $2.80 \pm 3.30(0.10 \pm 0.39)$ \\
Treponema denticola & $4.52 \pm 4.65(2.15 \pm 2.62)$ & $4.46 \pm 4.66(2.51 \pm 3.61)$ & $4.27 \pm 4.47^{*}(1.49 \pm 2.09)$ \\
Tannerella forsythia & $4.74 \pm 4.92(3.15 \pm 4.04)$ & $4.66 \pm 4.88(4.02 \pm 6.77)$ & $4.69 \pm 5.01^{*}(3.55 \pm 5.82)$ \\
Prevotella intermedia & $5.49 \pm 5.98(18.29 \pm 54.65)$ & $5.94 \pm 6.60(60.61 \pm 291.80)$ & $5.99 \pm 6.68(62.60 \pm 310.50)$ \\
\hline
\end{tabular}

Data shown as mean \pm standard deviation. ${ }^{*} \mathrm{p}<0.05,{ }^{* *} \mathrm{p}<0.01$ vs. baseline 


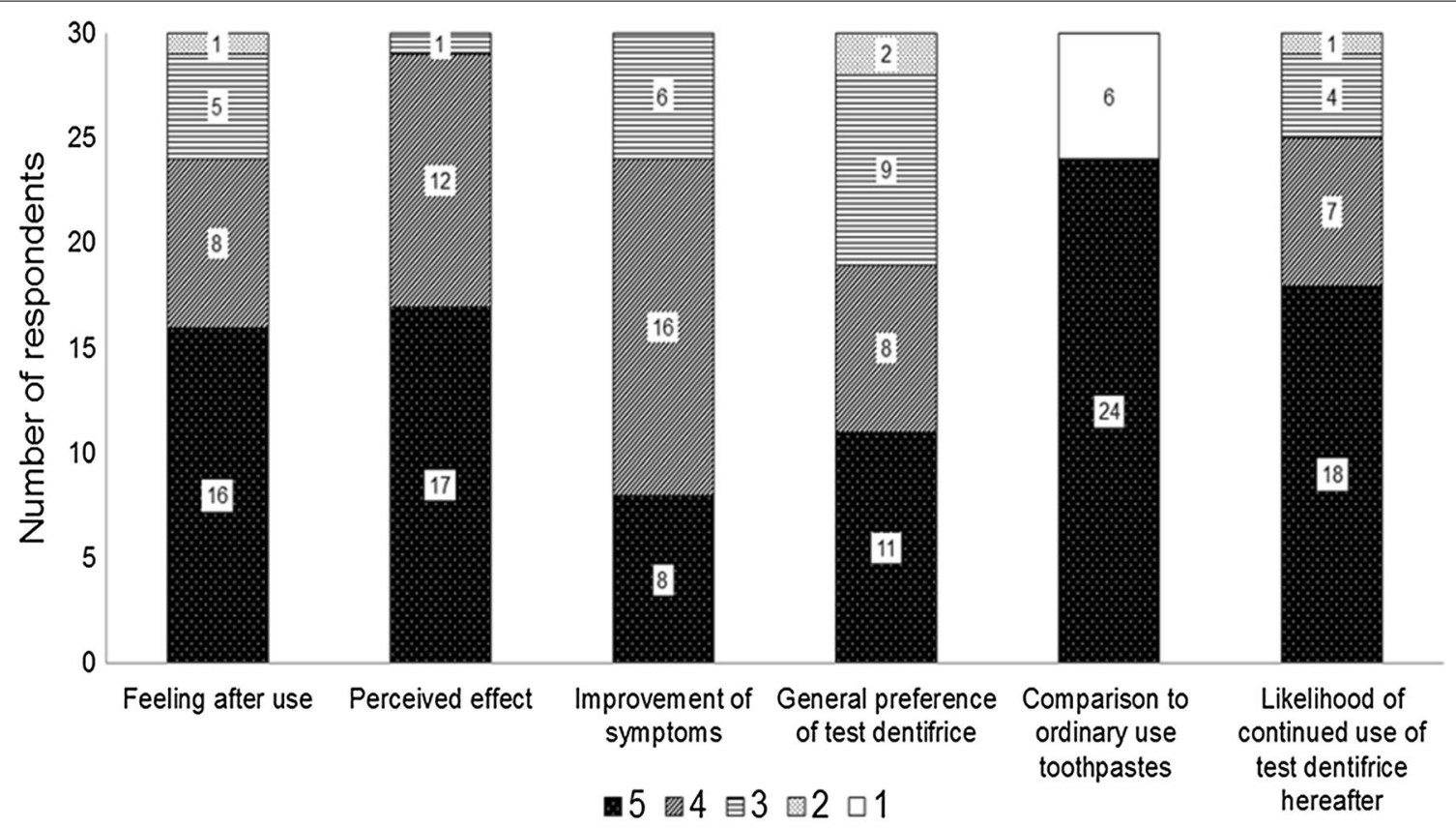

Questionnaire items

Fig. 1 Subjective evaluation by participants: distribution of responses

scored 2 (probably not). Overall, those who scored 4 or 5 accounted for $63 \%$ or more in questionnaire items.

\section{Discussion}

In the present study, we investigated the effect of the test dentifrice, which contains sodium lauroylsarcosine, isopropyl methylphenol and cetylpyridinium chloride and other medicinal ingredients, on periodontal conditions of patients undergoing SPT. Following the use of the test dentifrice for 4 weeks, significant improvements from baseline were observed in all periodontal parameters. No significant adverse events were noted. These findings suggest that this dentifrice can be safely used to prevent the progression of periodontitis during SPT. It has been reported that gingivitis is alleviated by using dentifrices containing $\beta$-glycyrrhetinic acid $[10,11], \varepsilon$-aminocaproic acid $[9,10]$ or sodium chloride $[9,10]$. Improvement in periodontal conditions observed in this study can be attributed to any of these and the bactericidal ingredients, because the test dentifrice contains all of them. In the subjective evaluation of overall usefulness, $60 \%$ of clinicians reported "mild improvement". This may be attributed to the fact that the periodontal conditions at baseline were relatively stable for these participants because they were already in the SPT phase.

As for the microbiological testing, we targeted the representative periodontal pathogens in saliva. Among them, $P$. gingivalis, $T$. denticola and $T$. forsythia belong to the 'red complex' bacterial species [12], and their close associations with periodontitis have been reported [13, 14 ]. In this study, the numbers of $T$. denticola and $T$. forsythia significantly decreased at 4 weeks compared to baseline. This finding suggests that the test dentifrice may exert a suppressing effect on the growth of the periodontal pathogens. Sodium lauroylsarcosine is an anionic surfactant [4]. Anionic surfactants have been shown to exert a bactericidal effect due to its ability of destroying bacterial cell membranes and denaturing bacterial proteins. Isopropyl methylphenol has been reported to penetrate biofilm and exert bactericidal action [15]. In an ex-vivo experiment, a mouthrinse containing $0.05 \%$ cetylpyridinium chloride demonstrated a significant antimicrobial activity on plaque microorganisms [16]. These ingredients in the test dentifrice might have contributed to suppressing the periodontal pathogens.

In the assessment of inflammatory markers in GCF, IL- $1 \beta$ was found to be significantly decreased at 2 weeks compared to baseline. This suggests that this dentifrice has a potential effect of suppressing gingival inflammation from the relatively early stage of use. An in vitro study showed that the production of IL- $1 \beta$ was decreased by dentifrices containing triclosan [17, 18]. However, the test dentifrice in the present study does not include triclosan. Information is limited on the relationship between the dentifrice ingredients and the dynamics of inflammatory markers in periodontal milieu. Further 
investigation is necessary for understanding the true effect of test dentifrice ingredients on the release of inflammatory markers.

With regard to the subjective evaluation of the use of test dentifrice by participants, their responses for items such as feeling of use (taste, smell), feeling of effect (sense of refreshing and its duration), or whether to use in the future, were generally favorable. On the other hand, regarding the improvement of the symptoms and preference of this product, the responses were somewhat different. It may have been difficult for the participants to be conscious of a potential change in their oral conditions, compared to patients who are undergoing active periodontal treatment. Furthermore, the reason for relatively poor score on the preference of the test dentifrice may be attributed to somewhat salty flavor of the test dentifrice.

\section{Conclusions}

Significant improvements were found in the periodontal parameters, microbiological findings in saliva and IL-1 $\beta$ in GCF, following the 4-week use of the novel dentifrice containing 3 kinds of bactericidal ingredients. Both clinicians' and participants' perceptions of the use of the test dentifrice were generally favorable. The data collectively suggest that the progression of periodontal disease during SPT may be prevented by the use of this dentifrice.

\section{Limitations}

This was a single-arm, prospective, non-randomized study with no control group. Since the sample size is small, it is difficult to draw definite conclusions or broad generalizations from this study. It is also difficult to interpret how subjective the clinician and patient reporting is.

A randomized controlled trial using a larger sample size is necessary to clarify the true clinical benefit of the test dentifrice.

\section{Additional files}

Additional file 1: Table S1. Subjective evaluation by participants: Questionnaire items.

Additional file 2: Table S2-1. The amounts of inflammatory markers in GCF (pg/mL). Table S2-2. The amounts of each inflammatory markers to 1 $\mathrm{mg}$ total protein $(\mathrm{pg} / \mathrm{mg})$.

\section{Abbreviations}

SPT: supportive periodontal therapy; PD: probing depth; Gl: gingival index; PII: plaque index; CAL: clinical attachment level; GCF: gingival crevicular fluid.

\section{Authors' contributions}

DK and TK contributed to study conception and design, analyzed and interpreted data, and drafted the manuscript. AY, MT, ME, AM-O, ST and AS contributed to study conception and design. ME, AM-O, ST and AS contributed to data acquisition and revised the manuscript. All authors read and approved the final manuscript.

\section{Author details}

${ }^{1}$ Department of Periodontology, Tokyo Dental College, Tokyo, Japan. ${ }^{2}$ Kinumatsu Dental Clinic, Chiba, Japan. ${ }^{3}$ Daiichi Sankyo Healthcare Co., Ltd., Tokyo, Japan. ${ }^{4}$ Oral Health Science Center, Tokyo Dental College, Tokyo, Japan.

\section{Acknowledgements}

The authors are grateful to all patients who participated in this study.

\section{Competing interests}

This study was concluded as contract research with Daiichi Sankyo Healthcare Co., Ltd., Tokyo, Japan. The company provided us with research expenses, dentifrice and toothbrush. Authors Atsushi Yokomizo and Miki Tanaka work for Daiichi Sankyo Healthcare Co., Ltd., Tokyo, Japan, and they were not involved in data analysis and interpretation. The authors received no commercial input in the preparation of the manuscript.

\section{Availability of data and materials}

Data and further information about "Methods" section are available from the corresponding author.

\section{Consent for publication}

Not applicable.

\section{Ethics approval and consent to participant}

This study was conducted with the approval of the Tokyo Dental College Ethics Review Committee (No. 697). All participants signed a written informed consent.

\section{Funding}

This study was funded by Daiichi Sankyo Healthcare Co., Ltd, Tokyo, Japan. The authors had full control of the data, of the analyses and received no commercial input in the preparation of the manuscript.

\section{Publisher's Note}

Springer Nature remains neutral with regard to jurisdictional claims in published maps and institutional affiliations.

Received: 27 November 2017 Accepted: 1 February 2018

Published online: 09 February 2018

\section{References}

1. Aida J, Ando Y, Akhter R, Aoyama H, Masui M, Morita M. Reasons for permanent tooth extractions in Japan. J Epidemiol. 2006;16:214-9.

2. Pihlstrom BL, Michalowicz BS, Johnson NW. Periodontal diseases. Lancet. 2005;366:1809-20.

3. Morris AJ, Steele J, White DA. The oral cleanliness and periodontal health of UK adults in 1998. Br Dent J. 2001;191:186-92.

4. Numabe Y. Consideration to the effects of dentifrice on Periodontal disease. J J.pn Soc Periodontol. 2014;56:259-66 (in Japanese).

5. Davies RM. Toothpaste in the control of plaque/gingivitis and periodontitis. Periodontol 2000. 2008:48:23-30.

6. Armitage GC. Development of a classification system for periodontal diseases and conditions. Ann Periodontol. 1999;4:1-6.

7. Hisano A, Sato S, Kamoi H, Takayanagi K, Ito A, Ito T, et al. Clinical effect of dentifrice containing sodium ascorbate on periodontitis. J Jpn Soc Periodontol. 2004;46:137-42 (in Japanese).

8. Irokawa D, Takeuchi T, Noda K, Goto H, Egawa M, Tomita S, et al. Clinical outcome of periodontal regenerative therapy using collagen membrane and deproteinized bovine bone mineral: a 2.5-year follow-up study. BMC Res Notes. 2017;10:102

9. Watanabe Y, Miyata T, Kusunoki K, Hikima T, Ikeda K, Yamazaki Y, et al. Clinial effects of dentifrices containing sodium chloride and anti-inflammatory agents on periodontal disease. Oral Ther Pharmacol. 1984;3:16473 (in Japanese). 
10. Watanabe Y, Fujihashi H, Hiyoshi K, Miwa Y, Kusunoki K, Hikima T, et al. Clinical effects of dentifrice containing sodium chloride and anti-inflammatory agents. J Jpn Soc Periodontol. 1982;24:299-308 (in Japanese).

11. Nakao S, Yasui T, Tanaka S, Onozawa H, Tanaka S, Ootaka Y, et al. Clinical evaluation of the dentifrice containing tranexamic acid and dipotassium glycyrrhizinate for periodontal disease. J Dent Health. 1991;41:643-53 (in Japanese).

12. Socransky SS, Haffajee AD, Cugini MA, Smith C, Kent RL Jr. Microbial complexes in subgingival plaque. J Clin Periodontol. 1998;25:134-44.

13. Darveau RP. Periodontitis: a polymicrobial disruption of host homeostasis. Nat Rev Microbiol. 2010;8:481-90.

14. Holt SC, Ebersole JL. Porphyromonas gingivalis, Treponema denticola, and Tannerella forsythia: the "red complex", a prototype polybacterial pathogenic consortium in periodontitis. Periodontol 2000. 2005;38:72-122.
15. Kadoya H. Prevalence of periodontal diseases and development of oralcare products for prevention of the diseases. Fragr J. 2004;32:61-6 (in Japanese)

16. Sreenivasan PK, Haraszthy VI, Zambon JJ. Antimicrobial efficacy of $0.05 \%$ cetylpyridinium chloride mouthrinses. Lett Appl Microbiol. 2013;56:14-20

17. Mustafa M, Wondimu $B$, Ibrahim M, Modéer T. Effect of triclosan on interleukin-1 beta production in human gingival fibroblasts challenged with tumor necrosis factor alpha. Eur J Oral Sci. 1998;106:637-43.

18. Modéer T, Bengtsson A, Rölla F. Triclosan reduces prostaglandin biosynthesis in human gingival fibroblasts challenged with interleukin-1 in vitro. J Clin Periodontol. 1996;23:927-33.

\section{Submit your next manuscript to BioMed Central and we will help you at every step:}

- We accept pre-submission inquiries

- Our selector tool helps you to find the most relevant journal

- We provide round the clock customer support

- Convenient online submission

- Thorough peer review

- Inclusion in PubMed and all major indexing services

- Maximum visibility for your research

Submit your manuscript at www.biomedcentral com/submit 